\title{
MEASUREMENTS OF SURFACE KINEMATICS AND TEMPERATURE IN THE SURF AND SWASH ZONES USING INFRARED IMAGE VELOCIMETRY
}

\author{
Zhi-Cheng Huang ${ }^{1}$, Kao-Shu Hwang², Luc Lenain³ ${ }^{3}$ W. Kendall Melville³, Hwung-Hwang \\ Hwung $^{4}$
}

\begin{abstract}
High intensity air bubbles generated in the surf zone and the thinning of swash flow make velocity measurements particularly challenging in coastal areas. These facts have led the need for a new measurement technique to quantify the surf and swash flow dynamics. Here, we tested infrared image techniques to measure the surface temperature and then to derive the velocity fields using cross-correlation algorithm for large-scale solitary waves breaking in the surf and swash zones. From the comparison with unspiked electromagnetic current meter (EMCM) data and previous validation, it is suggested that the infrared image velocimetry (IRIV) is satisfactory to quantify the surface turbulent flow in the surf and swash zones. The data obtained in the experiment provides a new description of surface thermal structure and kinematics for solitary breaking waves. Two-dimensional organized streaks of temperature structures are evident on the water surface behind the head of rebounding jet. Wavenumber spectrum analysis shows that the directionality of these thermal signatures evolves with time. Evolution of vorticity on the water surface during the run-up and run-down process of the solitary broken wave is discussed.
\end{abstract}

Keywords: Solitary wave; wave breaking; surf zone; swash zone; infrared

\section{INTRODUCTION}

Hydrodynamics in surf and swash zones plays an important role in sediment transport and structure stability in coastal areas. However, measurement of the flow velocity in natural surf and swash zones has been considered a challenge due to the occurrence of signal dropout caused by highintensity bubble entrainment. The thinning of run-up and run-down flow in swash zones also makes measurements particularly challenging with some in-situ velocimetry techniques (e.g., electricmagnetic current meter, EMCM, and acoustic Doppler velocimetry, ADV) with their weaknesses in natural surf and swash zones (Holland et al., 2001).

A common instrument for measurements of internal flow velocities in small laboratory surf zones is the laser Doppler velocimetry (LDV). LDV has been used to measure the flow structure, undertow, turbulence intensity, turbulent transport terms, and Reynolds stresses in small laboratory surf zones under spilling and plunging breakers (e.g., Stive, 1980; Nadaoka and Kondoh, 1982; Ting and Kirby, 1994; Ting and Kirby, 1995; Ting and Kirby, 1996; Stansby and Feng, 2005; De Serio and Mossa, 2006; Shin and Cox, 2006). However, measurements using LDV are usually restricted below wave trough level due to the failure of this technique (signal drop-out) in the aerated region of breaking waves. In the last decade, particle image velocimetry (PIV) has also been widely used in small laboratories for various types of breaking waves outside the aerated region or for small-scale breakers (e.g. Chang and Liu, 1999; Qiao and Duncan, 2001; Melville et al., 2002; Huang et al., 2009). When performing measurements using a typical PIV, air bubbles also make the laser sheet uncontrollable in the aerated region (Ryu et al., 2005) and the high-power laser light may be reflected by bubb1les and damages the charge-couple device (CCD). A new measurement technique- bubble image velocimetry developed by Ryu et al. (2005) assumes bubbles under breaking waves have a high traceability following to water fluid flow, may not be robust. In addition, recent progress of turbulence and current measurements in natural surf zones has been achieved using ADV (e.g., Ruessink, 2010; Feddersen, 2012). These measurements were also performed far from crest regions because air bubbles corrupt the acoustic signals, and data despiking and quality controls are essentially needed for ADV measurements in natural surf zones (Elgar et al., 2005; Feddersen, 2010).

Surface currents in the surf and swash zones have been measured using video and crosscorrelation based techniques (a PIV algorithm). Most of those methods rely on the advection of optically visible surface features, typically by determining the movement of bubbles and foam generated by breaking waves and bores, and by patches of light reflected from the water surface. However, the essential assumption adopted in this technique, which assumes these surface "particles" have a high traceability following to the surface flow, may not be robust. This has led to the need for a

\footnotetext{
${ }^{1}$ Graduate Institution of Hydrological and Oceanic Science, National Central University, Taoyuan 320, Taiwan.

2 Tainan Hydraulics Laboratory, Tainan 704, Taiwan.

${ }^{3}$ Scripps Institution of Oceanography, University of California San Diego, La Jolla, CA 92093-0213, USA.

${ }^{4}$ Department of Hydraulic and ocean engineering, National Cheng Kung University, Tainan 701, Taiwan.
} 


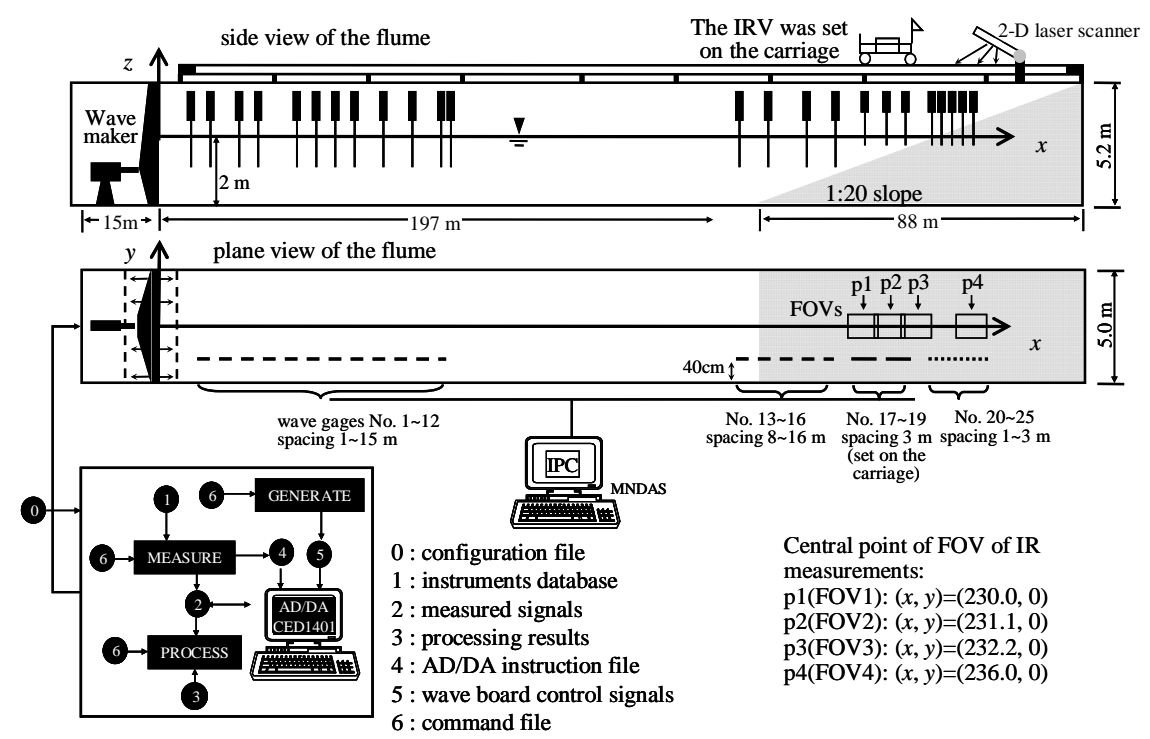

Figure 1. Schematic diagram of the experimental facilities and setup. A pc-controlled wavemaker and a fixed concrete sloping bottom were located in the left and right end of the flume. Total 25 wave gauges and an infrared image velocimetry (IRIV, set on the carriage) were used to measure the wave profiles and the surface temperature fields, respectively. The flow velocities were measured in the surf zone $\left(1^{\text {st }}\right.$ to $3^{\text {rd }}$ FOVs) and swash zone $\left(4^{\text {th }} \mathrm{FOV}\right)$ by moving the carriage and repeating the experiments with the same testing conditions.

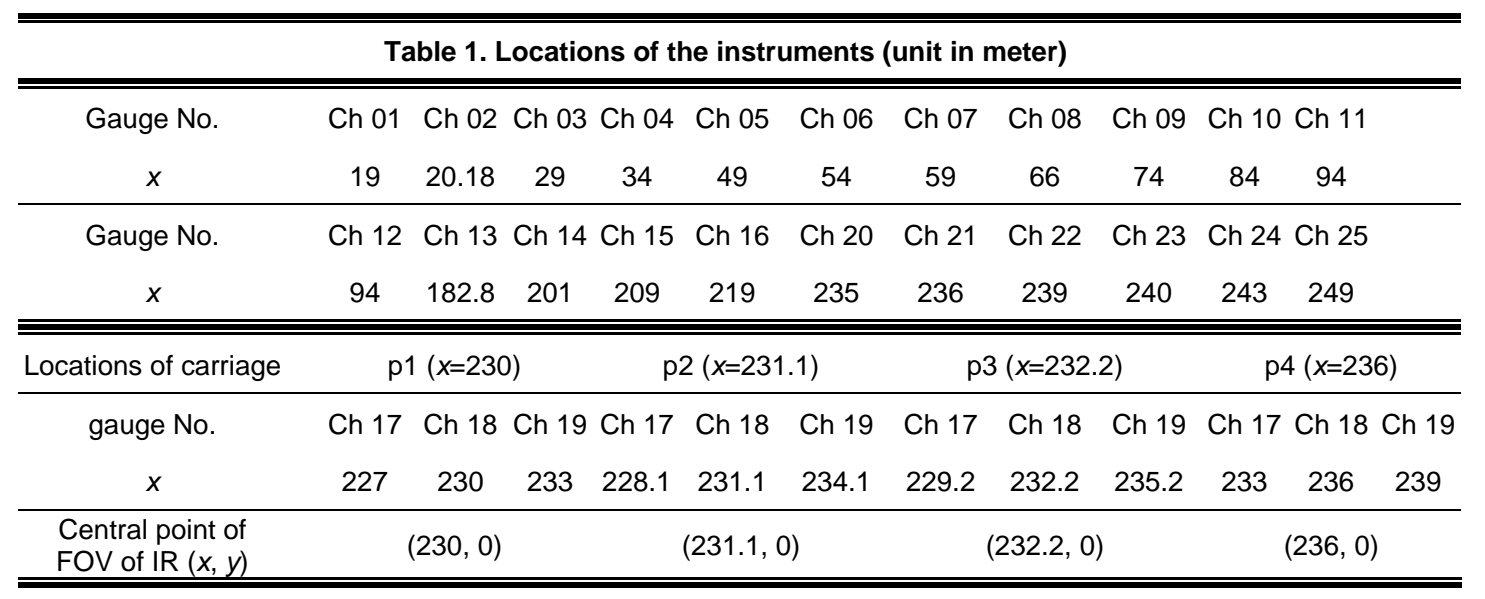

novel measurement technique to achieve high-quality surface flow measurements in the surf and swash zones.

Here, we tested infrared image techniques and the so called infrared image velocimetry (IRIV) to experimentally study large-scale solitary waves breaking in prototype-scale surf and swash zones. The measured infrared data are used to study the evolution of surface temperature structures and to compute the surface flow velocity and its spatial derivatives during solitary-wave breaking. New experimental results for the surface temperature structures and velocity fields of the solitary wave breaking in surf and swash zone are demonstrated.

\section{EXPERIMENTS}

\subsection{Experimental facilities and setup}

The experiments were conducted in the Super Tank of Tainan Hydraulics Laboratory (THL). The tank is $300 \mathrm{~m}$ long, $5.0 \mathrm{~m}$ wide and $5.2 \mathrm{~m}$ deep. Waves were generated by a programmable pccontrolled hydraulically driven, dry back, piston-type wavemaker at one end of the flume and a 1:20 concrete slope was constructed the tail of the flume at a distance of $197.0 \mathrm{~m}$ from the waveboard. A movable carriage was installed on rails on the sidewalls of the flume. Figure 1 shows the schematic diagram of the experimental facilities and setup and the defined Cartesian coordinate system ( $x$ : streamwise/along flume direction, $y$ : lateral/cross flume direction, and $z$ : vertical direction). 


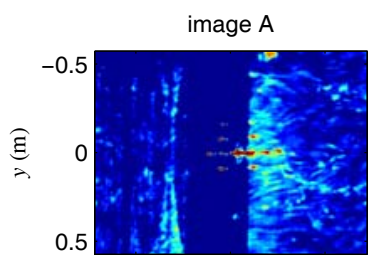

image B

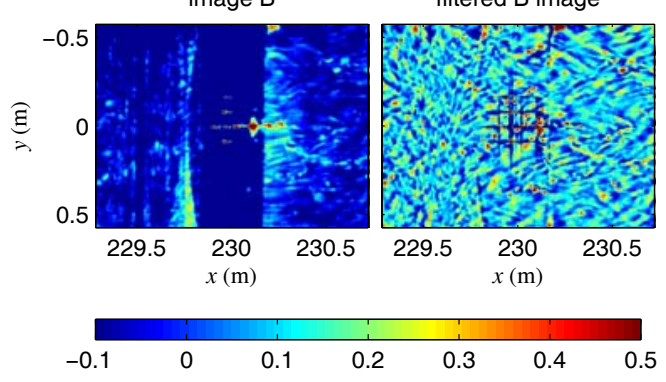

filtered $A$ image

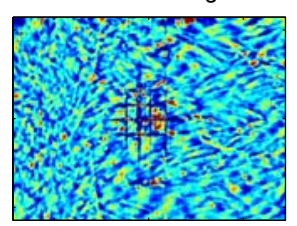

filtered B image
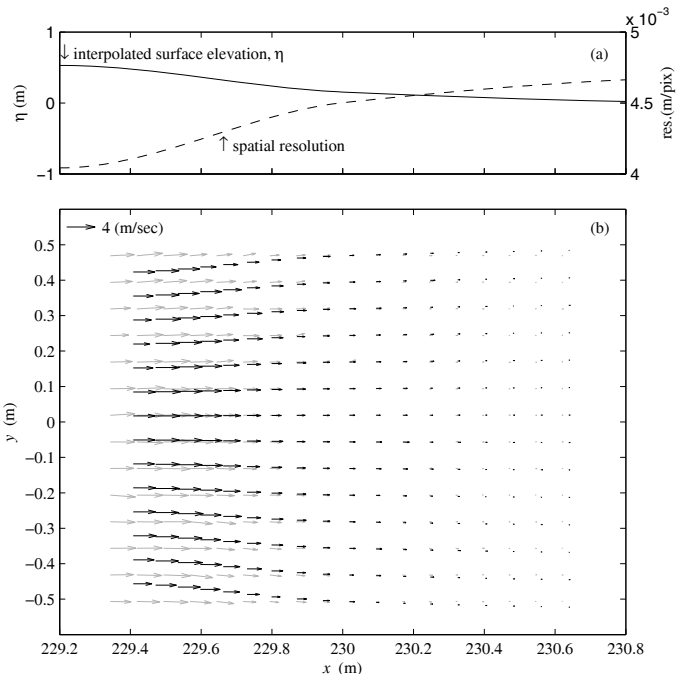

Figure 2. Example of velocity calculation from infrared image pair with cross-correlation algorithm. The four images in the left panels are the measured water surface temperatures related to the mean subskin temperature of $298.78 \mathrm{~K}$. The vertical cold/low temperature structures (dark blue) in the original images came from the reflection of the carriage metal bar, and they are removed by high-pass filtering before velocity calculation as shown in the filtered images. The right lower panel (b) below is the computed velocity vectors without (black) and with correction of varying spatial resolution (gray), which is obtained by prior calibration based on the spatial profile of surface elevation interpolated from wave gauge measurements and is shown in the right upper panel (a). Note that the velocities are displayed every other for ease observation.

The water surface elevations were measured by twenty-two capacitance-type wave gauges that were mounted $40 \mathrm{~cm}$ from the sidewall (No. 1 - 16 and No. 20 - 25) and by three ultra-sonic wave gauges that were mounted on the carriage (No. 17 - 19). The $18^{\text {th }}$ gauge was set along the negative $y$ direction outside the center of the field-of-view (FOV) boundary of the infrared image, and the $17^{\text {th }}$ and $19^{\text {th }}$ gauges were set three meters from the $18^{\text {th }}$ gauges along the $x$ direction. The ultra-sonic wave gauges were moved with the carriage to the four positions of the IRIV measurements (p1 - p4). The wave profiles were recorded simultaneously with a $50 \mathrm{~Hz}$ sampling rate using the Microsoft NT based Multi-Nodes-Data-Acquisition-System (MANDAS), developed by THL (Hwung and Chiang, 2005). The streamwise and vertical flow velocities were measured by an EMCM with a sampling rate of 50 $\mathrm{Hz}$, which was set close to the FOV boundary of the infrared image. The vertical positions of EMCMs were set at half of the local still water depth. The positions of the wave gauges and the EMCMs are summarized in Table 1.

The surface temperatures were recorded by an integrated infrared imaging system developed by Veron et al. (2008). This system includes an infrared digital camera (Indigo Merlin MWIR camera), a 60W air-cooled CO2 laser (Synrad Firestar T60) equipped with an industrial marking head (Synrad FH index) with two computer-controlled scanning mirrors. These instruments were mounted inside a weatherproof, air-conditioned aluminum housing. More details about the infrared imaging system can be found in Veron et al. (2008). The infrared camera, manufactured with a thermal resolution NETD of $18-\mathrm{mK}$, was set to measure the temperature images $(320 \times 256$ pixels) at $60 \mathrm{~Hz}$ at four FOVs by moving the carriage. The first to the third FOVs were overlapped to observe the overturning and impinging surface temperature of the solitary breaking wave in the surf zone. The fourth FOV is near the shoreline to observe the swash process of the turbulent bore. The positions of the four FOVs are summarized in Table 1.

In this study, solitary waves were generated using Goring's (1987) procedure. The free surface elevation, $\eta$, is described as

$$
\eta(x, t)=H \sec ^{2}\left[\sqrt{\frac{3 H}{4 h_{0}^{3}}}(x-C t)\right]=H \sec ^{2}(\theta),
$$



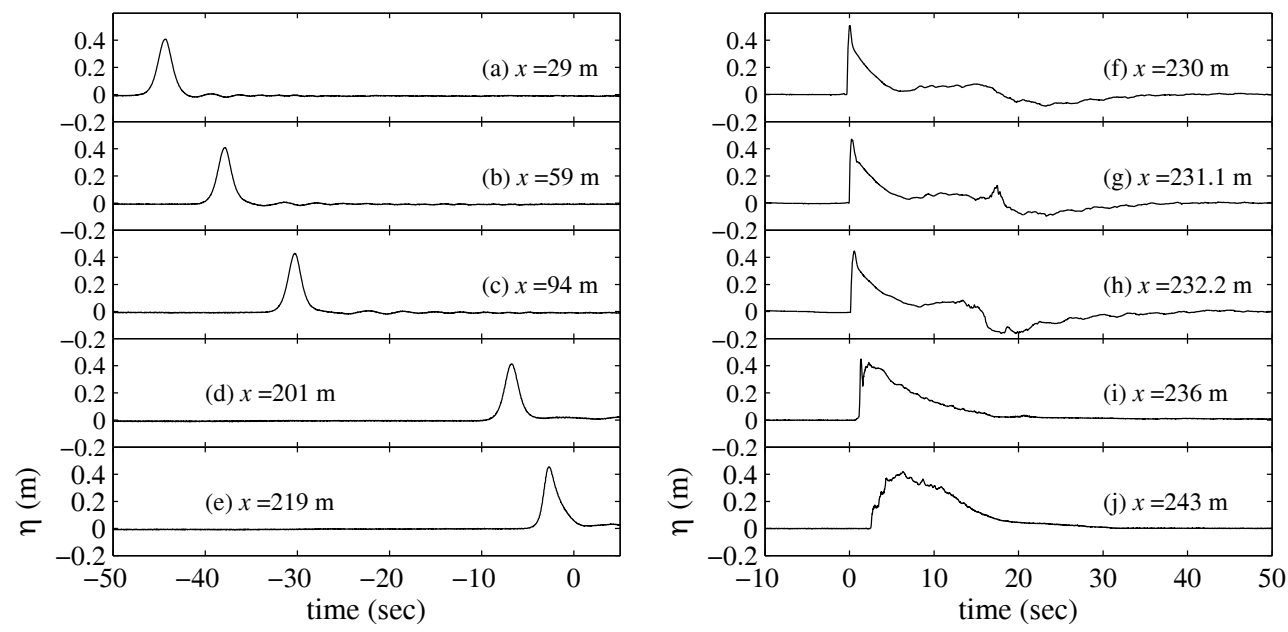

Figure 3. Time series of measured water surface elevations in the flume. Panels of (a-c) are in the constant water depth, (d-e) are in the shoaling region, (f-g) are at the locations of wave breaking during overturning, (h) is at the wave-breaking impinging point, and (i-j) are at the swash zone.

$$
C=\sqrt{g h_{0}\left(1+H / h_{0}\right)},
$$

where $H$ is the wave height, $h_{0}$ is the constant water depth, , $t$ is the time, $C$ is the phase speed, $\theta$ is the wave phase, and $g$ is the gravity acceleration. The solitary wave is generated with a height $H=41 \mathrm{~cm}$ in a water depth $h_{0}=2 \mathrm{~m}\left(H / h_{0}=0.205\right)$. The measured surface profile well fitted the theoretical solution (Hwang et al., 2007). We conducted the same experiments twice at each FOV by moving the carriage and the instruments. All the data sets were synchronized.

\subsection{Data analysis}

The recorded infrared images were analyzed to compute surface velocity fields using crosscorrelation techniques. The temperature contrast of thermal structures in the water surface skin layer is fine enough in the present experiment; therefore, we only adopted passive technique (cross-correlation method) to calculate the velocity fields without using the active technique (tracking the laser-heated thermal markers). See Veron et al. (2008) for details of the passive and active techniques. For some phases of wave overturning process, the water surface received cold temperature sinks on water surface due to the reflection of metal bars of the carriage (left panels in Figure 2). An idealized highpass filtering technique (Gonzalez and Woods, 2002) is used to remove the low wavenumber structures. The movements of thermal structures can thus be identified visually or using the cross-correlation algorithm.

Image pairs of varying straddled time intervals ranging from 1/60 to $1 / 3$ seconds were analyzed to compute the velocity using a cross-correlation algorithm. The varying time intervals were used due to the broad range of surface velocities. The time intervals were adjusted depending on the magnitude of surface current. The movements of thermal structures between image pairs were examined visually to ensure that the time interval was large enough to identify the displacement of temperature structures. The time intervals provide sufficient displacement to avoid errors arising from sub-pixel interpolation. Fast-Fourier-Transform-based normalized cross-correlation is applied in the analysis, similar to standard cross-correlation techniques (Raffel et al., 1998). The normalization compensates for uneven contrast in the sub-images. In a similar fashion to standard PIV routines, each pair of succeeding images was processed from an interrogation sub-image size of $16 \times 16$ pixels with $50 \%$ linear overlap and a step size of $8 \times 8$ pixels. A three-point Gaussian interpolation scheme was used for the sub-pixel accuracy of the peak on the correlation plane. The cross-correlation algorithm gives the average displacement between the two sub-images of the image pair, and the average displacement and time interval between the two sub-images gives the average velocity over the sub-image. Erroneous velocities and outliers are removed from the velocity field using a dynamic mean filter (Raffel et al., 1998; Veron et al., 2008). An example of the velocity computation is shown in Figure 2.

Because the FOVs of the infrared images are directly on the water surface, the FOV size and the spatial resolutions of images depend on the distance between the camera and the water surface, i.e. 

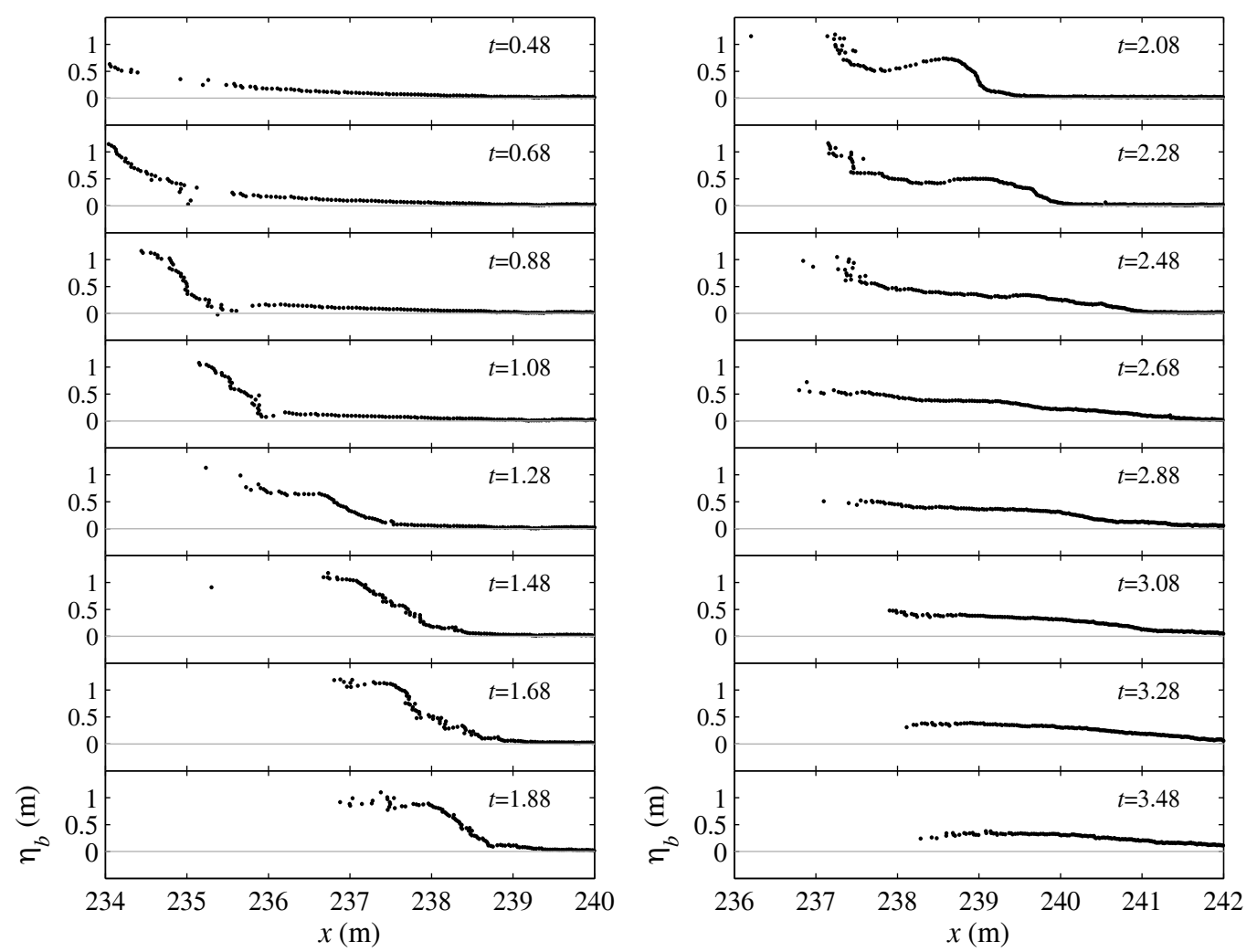

Figure 4. Evolution of surface elevation profiles of the solitary turbulent bore propagating in the swash zone measured by LIDAR. Note the coordinate of the $y$-axis was change to that on the sloping bottom.

spatial resolutions vary with the surface elevation. Varying spatial resolution being functional to the surface elevation is required to convert the particle displacements in image dimensions to real physical quantities. The spatial resolution is computed from a prior calibration on a relation of the distance between the camera and the surface elevation. Ideally, a 2-D surface elevation map in $x-y$ (streamwise and transverse) plane is needed for the conversion. In the almost 2-D flume experiment, the variation of the resolution in the $y$ direction was ignored and the corrections only require spatial profile of surface elevations in the $x$-direction. Since the $18^{\text {th }}$ wave gauge was set at the center of each FOV, the surface elevation measured by this wave gauge provides unbiased spatial resolution at the central point of images. However, spatial profile of the surface elevation is still needed to correct the varying resolution for the entire FOV. The synchronized surface elevation data recorded at the 11 gauge stations near the IRIV measurements were used to obtain the spatial profiles of surface elevation using a cubic spline interpolation scheme. The coordinates and magnitudes of the spatially distributed velocities were corrected by the varying spatial resolution. Note that some detailed local structure of the breaking wave, such as surface rollers and water splashing, may be smoothed by the spline interpolation. The right panels of Figure $\mathbf{2}$ shows an example of the interpolated spatial surface profile, its corresponding spatial resolution of the infrared images and uncorrected and corrected velocity vectors. Variations between the uncorrected and corrected velocity vectors can be seen when the difference of surface elevation to the central of the FOV increases.

\section{RESULTS}

\subsection{Wave profiles}

Figure 3 presents time series of mean surface elevations measured by wave gauges. The mean profiles were obtained by averaging eight synchronized test runs. The standard deviations are all about $1 \mathrm{~cm}(\sim 2.4 \%$ of the incident wave height) before wave breaking. The value is about $10 \mathrm{~cm}$ at the extreme steep front wave crest and is about $1-2 \mathrm{~cm}$ after wave breaking. It is seen that the wave maintains its symmetric shape with respect to the center of the wave crest in the constant water depth ( $x=0 \sim 197 \mathrm{~m})$. It shoals on the slope $(x=197 \sim 230 \mathrm{~m})$ and gradually transforms into a triangle shape with steeping front crest and gentle rear face until breaking occurs. The wave breaking begins 

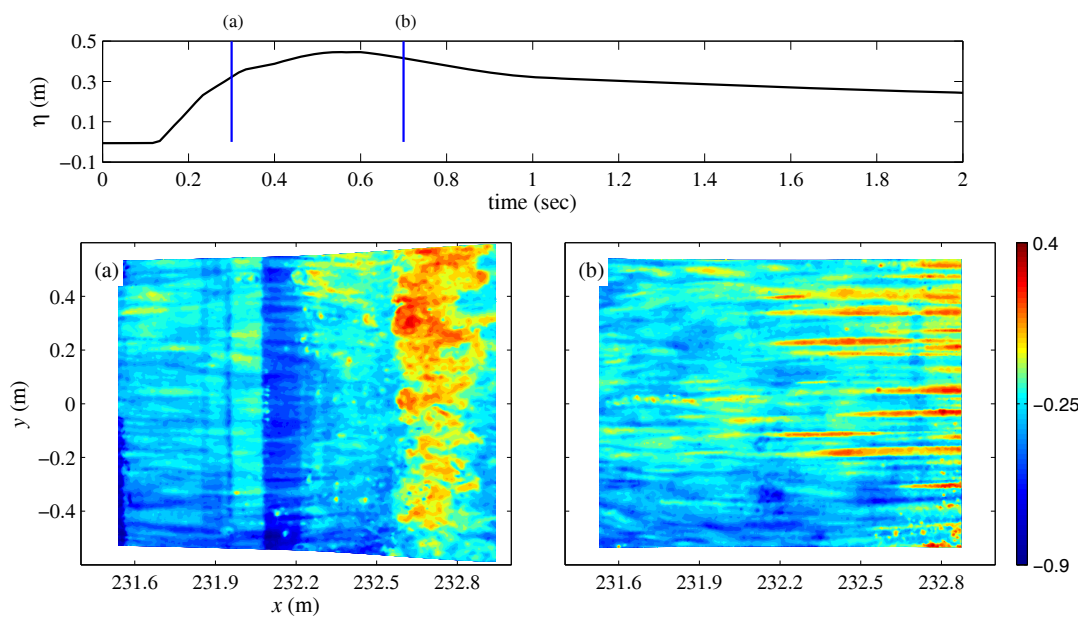

Figure 5. Surface thermal structure at the impinging point (FOV3) of the solitary breaking wave in the surf zone. The upper panel is the time series of surface elevation measured at $x=232.2 \mathrm{~m}$. The panels (a-b) are the surface temperature fields relative to the mean subskin temperature $298.03 \mathrm{~K}$ at (a) the impact point of a solitary breaking wave and (b) 0.4 seconds later. The time stamps of the snapshots $(a-b)$ are marked as the blue vertical lines in the upper panel. Note the warm patches (red color) in (a) are the head of the turbulent bore.

with the formation of an overturning jet at about $x=230 \mathrm{~m}$ (FOV1), which is defined as the breaking point, and the moment of the maximum elevation at this site is defined as $t=0 \mathrm{sec}$. The plunging jet projects into the water surface ahead at about $x=232 \mathrm{~m}$ (FOV3), which is defined as the impinging point, and then the splashing water mixes with air bubbles to form a rebounding jet of two-phase foamy flow shoreward. The wave turns into a turbulent bore and propagates toward the shoreline $(x=$ $236 \mathrm{~m})$.

Figure 4 shows the evolution of the surface elevation profiles in the swash zone measured by a light detection and ranging technique (LIDAR). Near the shoreline $(x=236 \mathrm{~m})$ during $t=0.88-1.88$ $\mathrm{sec}$, the height of the turbulent bore is about $1 \mathrm{~m}$ and the width of the bore head is about 1-1.2 $\mathrm{m}$, resulting a remarkable slope of approximately $0.83-1$. The surface elevation (potential energy) at $x=$ $238 \mathrm{~m}$ increases with time for $t<1.88 \mathrm{sec}$ but decreases with time when $t>1.88 \mathrm{sec}$. The change of sign occurs when the turbulent bore starts to 'deform' and becomes a 'tongue' shape to push the thinning water column ahead with decreasing water mass shoreward. The slope of the bore head dramatically decreases at this moment after it becomes a tongue shape and changes to a thin layer of run-up water mass in the swash zone.

\subsection{Surface temperature fields}

No obvious thermal signature variations were observed in locations seaward of the plunging point, indicating negligible disturbances to disrupt the skin layer in this region. Remarkably warmer patches were observed at the head of the bore after the plunging jet projected into the water surface ahead to form a turbulent bore (left panel in Figure 5). In particular, several streaks of warmer temperature than the surroundings start to organize behind the head of the bore as shown in the right panel of Figure 5. These streaks are in the longitudinal direction, which is perpendicular to the wave crest line. They continue to strengthen, then weaken, and become isotropic as they return to the surrounding bulk temperature when the bore propagates further downstream.

Figure 6 shows two-dimensional wavenumber spectra of the surface temperature in FOV 3 corresponding to Figure 5. The shape of the corresponding surface temperature wavenumber spectrum starts to evolve in one preferred direction after the wave roller plunges ahead. The spectrum appears to be quite narrow and to centralize in one direction, indicating that the temperature streaks are aligned in the longitudinal direction. After the bore passes, the spectrum develops multidirectional and more isotropic shapes. Turbulences generated by a breaking wave disturb the skin layer at the water surface and transport warmer water from below (bulk or subskin water) to the free surface, resulting in cool and warm patches in infrared images. Therefore, the surface temperature signatures are believed to be associated with the status of turbulence generation at the skin layer (Jessup et al., 1997a; Jessup et al., 1997b; Loewen and Siddiqui, 2006; Watanabe and Mori, 2008). The anisotropy of the thermal structure may be caused from spanwise counter-rotating vortices behind the head of the bore. Multiple pairs of two-dimensional spanwise subsurface counter-rotating vortices produced behind the head of 


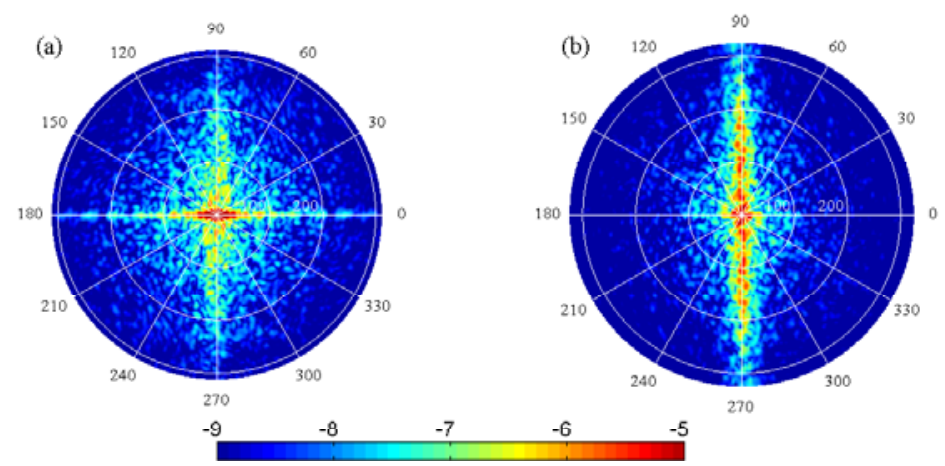

Figure 6. Two-dimensional wavenumber spectra of the surface temperature signatures plotted in cylindrical coordinates (unit in $\log \left(\mathrm{K}^{2} \mathrm{~m}^{2}\right)$ ) corresponding to Figure 5 . Note the direction of $0-180$ degree is the streamwise (along the flume) direction while $\mathbf{9 0 - 2 7 0}$ degree is the cross wave direction (cross the flume).
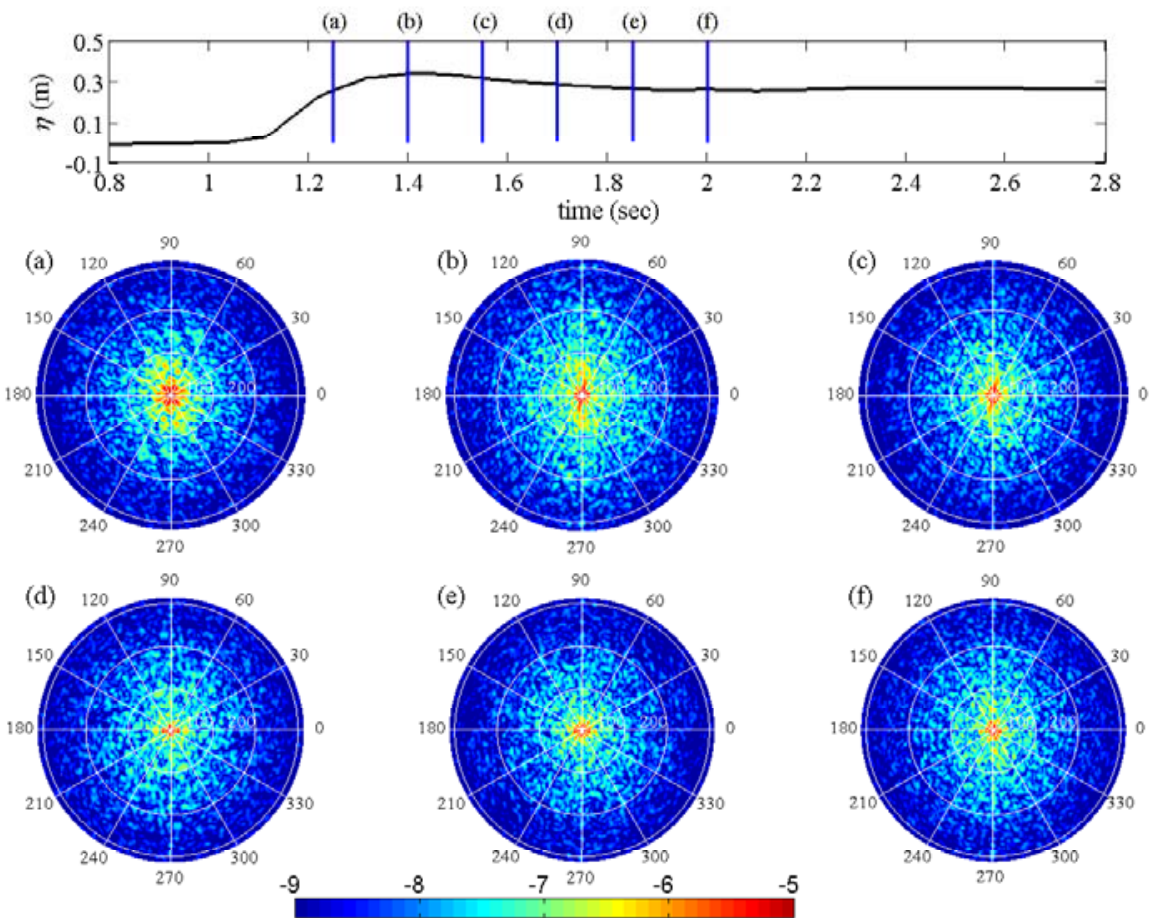

Figure 7. Two-dimensional wavenumber spectra of the surface temperature fields plotted in cylindrical coordinates (unit in $\log \left(K^{2} m^{2}\right)$ ) in the swash zone. The time stamps of the snapshots $(a-f)$ are marked as the blue vertical lines in the upper panel.

the bore may disturb the skin layer and transport warmer water from the subskin layer, resulting in the formation of well organized streaks aligned in the longitudinal direction. Then, the two-dimensional vortices may evolve to three-dimensional ones from the surface to the internal water column (Watanabe et al., 2005; Ting, 2008; Huang et al., 2010), causing the surface temperature distribution to become more isotropic.

Figure 7 shows the wavenumber spectra of the surface temperature in the swash zone (FOV 4). The shapes of the spectra in the swash zone all appear to have multidirectional and isotropic distributions during the inundation of the run-up bore and run-down flow. This confirms a homogeneous distribution of surface temperature fields. In addition, the surface temperature in the swash zone is much warmer than that in the surf zone. The isotropic and warmer temperature fields reflect the high-density air-bubble entrainment and three-dimensional bore turbulences in the swash flow.

\subsection{Surface velocity fields}

The surface velocities measured by the IRIV are compared with the internal flow velocities measured by EMCM as shown in Figure 8. For such a long wave, the flow velocity is usually assumed nearly uniform throughout the water column (Dean and Dalrymple, 1991). However, we found that 

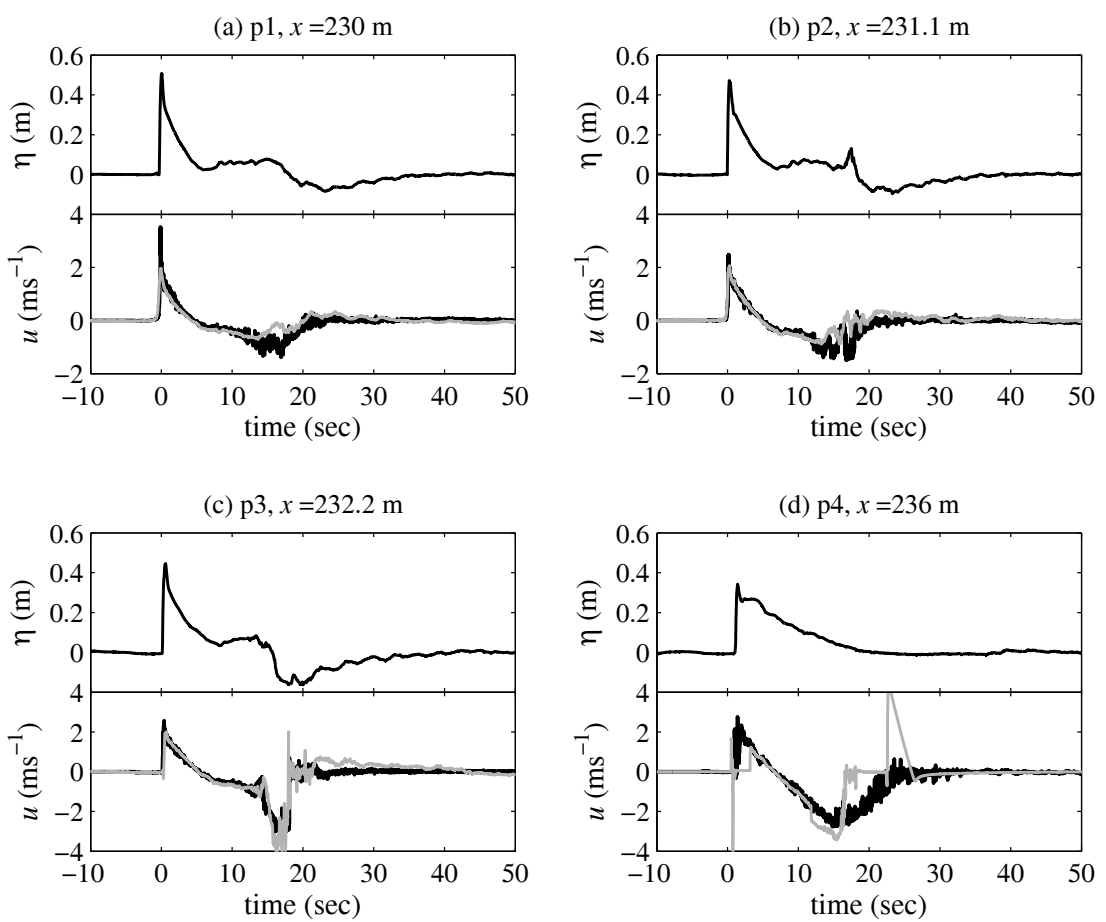

Figure 8. Comparisons of the measured time series of surface streamwise velocities $u$ by infrared image velocimetry (black lines) and by electric-magnetic current meter (gray lines) at the four FOVs. Note that the sampling points of the IRV and EMCM are at the same streamwise and lateral positions but at different vertical positions. Spikes of EMCM data are observed at p4 (swash zone).

the maximum surface streamwise velocity measured by IRIV is about 1.8 times the velocity measured by EMCM at the middle of the water depth at breaking location. Apart from the peaks of the crests at the wave breaking point, it is clear that the velocity magnitudes measured by the IRIV approximate those from the EMCM, confirming a reasonable assumption of depth-uniform on velocity. The agreement of the measured velocities between the IRIV and EMCM indicates the ability of this technique to quantify the surface flow in the surf and swash zone. However, signal spikes of the EMCM data are observed in the established-bore region (FOV3) and the swash zone (FOV4). The layer of the rundown flow is very thin and approaching zero during the rundown process ( $\mathrm{t}>15 \mathrm{sec}$, Figure 8d) and this EMCM is not capable to measure the extremely thin dun-down flow. This directly indicates the need for a new measurement technique to quantify such a complex two-phase flow in the surf and swash zone, and the IRIV is a satisfactory tool to measure the surface flow in the challenging coastal area.

An advantage of the IRIV is its ability to measure 2-D maps of surface velocities while the EMCM is a single-point current meter that can only sample the internal velocities at one fixed point. Figure 9 shows mean square vorticity of the measured surface velocities in the surf and swash zones, as a function of time and distance along the flume. Note the high and concentrated values of vorticity propagating onshore accompanying the movement of the bore head during $t \sim 0-3 \mathrm{~s}$. The vorticity generated in the breaking region comes from the rolling, plunging and splashing process. The vorticity is spread and advected to be extensively distributed in the swash zone, corresponding to the homogeneous and isotropic distribution of the temperature field. During the backwash process, a longer duration of vorticity generation is observed in the swash zone than in the surf zone and significant vorticities only occur in the established-bore region, further decreasing offshore.

\section{SUMMARY AND CONCLUSIONS}

Considering the instrument size and configuration, we setup an EMCM to measure the flow velocity in the surf zone and in the extremely thin layer of swash flow. In the experiments, signal spikes of the EMCM data are observed in the established-bore region in the surf zone and in the swash zone due to the high-intensity bubble entrainment and thinning of the flow layer. This directly indicates the need for novel measurement techniques to quantify such a complex two-phase flow. 


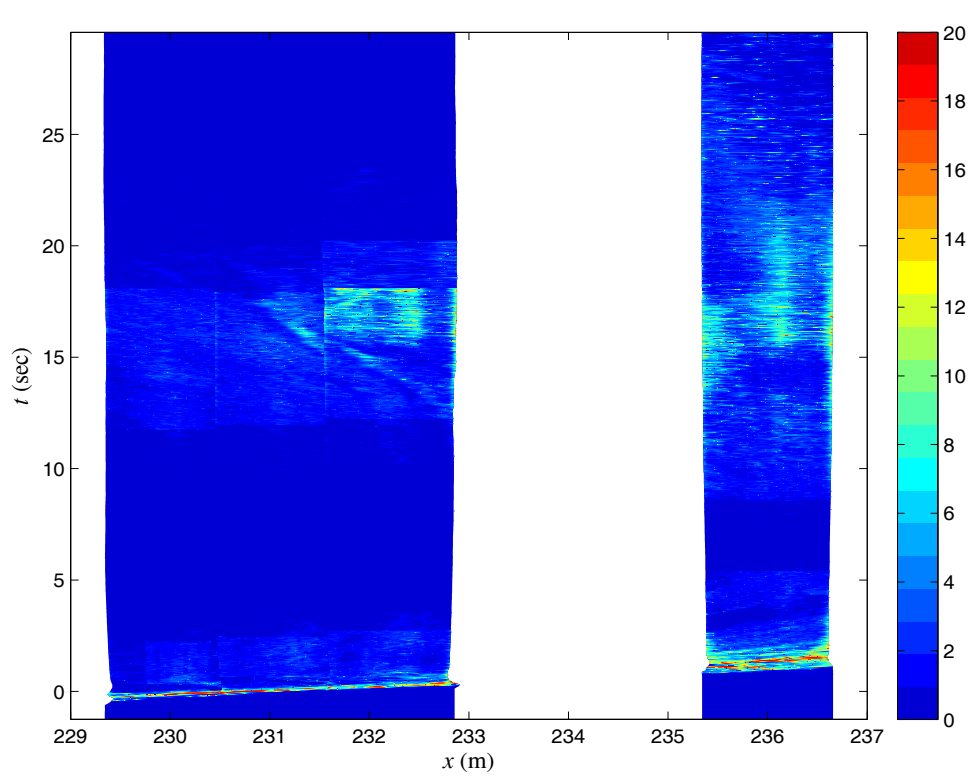

Figure 9. $x-t$ diagram of the mean square vorticity obtained by averaging the square vorticities in the $y$ (spanwise) direction.

Infrared image techniques were successfully used to measure the surface temperature fields of large-scale solitary waves breaking in the surf and swash zones. The temperature contrast of the water skin layer under the breaking wave is fine enough (by the $18-\mathrm{mK}$ resolution infrared camera) to compute the surface velocity fields using a cross-correlation algorithm. High-pass filtering, varying time intervals of image pairs and varying spatial resolutions are key factors to reduce errors during the velocity calculation. Except for the crest region at the breaking point and the spikes of the EMCM data, the surface flow velocity magnitudes measured by the IRIV approximate the flow velocity measured by the EMCM. From the agreement between the IRIV and EMCM velocity data and the previous validation done by Veron et al. (2008), it is suggested that the IRIV is a useful tool to quantify the surface turbulent flow in the surf and swash zone.

The data obtained during the testing and deployment of this instrumentation provides a novel description of surface thermal structure the kinematics under the solitary wave breaking. Twodimensional organized streaks of temperature structures are evident behind the head of the rebounding jet. The streaks are well-aligned in the longitudinal direction opposite to wave propagation. The streaks strengthen, decay, and develop isotropic to return to the surrounding bulk temperature during bore propagation. The shapes of the wavenumber spectra of the temperature fields in the swash zone all appear to have multidirectional and isotropic distributions during the inundation of the run-up bore and run-down flow. In addition, the 2-D maps of the surface velocities measured by the IRIV can provide spatial velocity derivates such as the vorticity fields. High and concentrated values of vorticity generation were evident in the crest region accompanying the movement of the head of the bore during the up-rush of the solitary breaking wave, while a longer duration of vorticity generation is observed during the backwash process.

\section{ACKNOWLEDGMENTS}

The authors would like to express sincere gratitude to the Research Center of Ocean Environment and Technology, National Cheng Kung University, and National Science Council (NSC) in Taiwan for their financial support (Grant No: NSC 97-2221-E-006-259-MY3). They would also like to thank the staffs in the Tainan Hydraulics Laboratory and Marine Physical Laboratory, Scripps Institution of Oceanography, for offering the facilities and conducting the experiments. We thank Prof. Herchang Ay for lending the infrared camera. ZCH was supported by NSC in Taiwan under a grant number: NSC 100-2119-M-003 -004.

\section{REFERENCES}

Chang, K.A. and Liu, P.L.F. 1999. Experimental investigation of turbulence generated by breaking waves in water of intermediate depth, Physics of Fluids, 11(11), 3390-3400. 
De Serio, F. and Mossa, M. 2006. Experimental study on the hydrodynamics of regular breaking waves, Coastal Engineering, 53, 99-113.

Dean, R.G. and Dalrymple, R.A. 1991. Water wave mechanics for engineers and scientists, Advanced Series on Ocean Engineering, 2. World Scientific.

Elgar, S., Raubenheimer, B. and Guza, R.T. 2005. Quality control of acoustic Doppler velocimeter data in the surf zone, Measurement Science \& Technology, 16, 1889-1893.

Feddersen, F. 2010. Quality Controlling Surf Zone Acoustic Doppler Velocimeter Observations to Estimate the Turbulent Dissipation Rate, Journal of Atmospheric and Oceanic Technology, 27(12), 2039-2055.

Feddersen, F. 2012. Observations of the Surf-Zone Turbulent Dissipation Rate, Journal of Physical Oceanography, 42(3), 386-399.

Gonzalez, R.C. and Woods, R.E. 2002. Digital image processing. Prentice Hall.

Goring, D.G., 1987. Tsunami: the propagation of long waves on a shelf, M. Keck. laboratory of hydraulics and water resources. California Institute of Technology, Pasadena, CA.

Huang, Z.C., Hsiao, S.C. and Hwung, H.H. 2009. Observation of coherent turbulent structure under breaking waves, International Journal of Offshore and Polar Engineering, 19(1), 15-22.

Huang, Z.C., Hwung, H.H. and Chang, K.A. 2010. Wavelet based vortical structure detection and length scale estimate for laboratory spilling waves, Coastal Engineering, 57, 795-877.

Hwang, K.S., Chang, Y.H., Hwung, H.H. and Li, Y.S. 2007. Large scale experiments on evolution and run-up of breaking solitary waves, Journal Earthquake and Tsunami, 1, 257-272.

Hwung, H.H. and Chiang, W.S. 2005. Measurements of wave modulation and breaking, Measurement Science \& Technology, 16, 1921-1928.

Jessup, A.T., Zappa, C.J., Loewen, M.R. and Hesany, V. 1997a. Infrared remote sensing of breaking waves, Nature, 385, 52-55.

Jessup, A.T., Zappa, C.J. and Yeh, J.H. 1997b. Defining and quantifying microscale breaking with infrared imagery., Journal of Geophysical Research-Oceans, 102, 23145-23153.

Loewen, M.R. and Siddiqui, M.H.K. 2006. Detecting microscale breaking waves, Measurement Science \& Technology, 17(4), 771-780.

Melville, W.K., Veron, F. and White, C.J. 2002. The velocity field under breaking waves: coherent structures and turbulence, Journal of Fluid Mechanics, 454, 203-233.

Nadaoka, K. and Kondoh, T. 1982. Laboratory measurement of velocity field structure in the surf zone by LDV, Coastal Engineering in Japan, 25, 125-146.

Qiao, H. and Duncan, J.H. 2001. Gentle spilling breakers: crest flow-field evolution, Journal of Fluid Mechanics, 439, 57-85.

Raffel, M., Willert, C.E. and Kompenhans, J. 1998. Particle image velocimetry. Springer.

Ruessink, B.G. 2010. Observations of Turbulence within a Natural Surf Zone, Journal of Physical Oceanography, 40(12), 2696-2712.

Ryu, Y., Chang, K.A. and Lim, H.J. 2005. Use of bubble image velocimetry for measurement of plunging wave impinging on structure and associated greenwater, Measurement Science \& Technology, 16, 1945-1953.

Shin, S. and Cox, D.T. 2006. Laboratory observations of inner surf and swash-zone hydrodynamics on a steep slope, Continental Shelf Research, 26, 561-573.

Stansby, P.K. and Feng, T. 2005. Kinematics and depth-integrated terms in surf zone waves from laboratory measurement, Journal of Fluid Mechanics, 529, 279-310.

Stive, M.J.F., 1980. Velocity and pressure field in spilling breakers, 17th Int. Conf. Coastal Eng. , pp. 547-566.

Ting, F.C.K. 2008. Large-scale turbulence under a solitary wave: Part 2 Forms and evolution of coherent structures, Coastal Engineering, 55, 522-536.

Ting, F.C.K. and Kirby, J.T. 1994. Observation of undertow and turbulence in a laboratory surf zone, Coastal Engineering, 24, 51-80.

Ting, F.C.K. and Kirby, J.T. 1995. Dynamics of surf zone turbulence in a strong plunging breaker, Coastal Engineering, 24, 177-204.

Ting, F.C.K. and Kirby, J.T. 1996. Dynamics of surf zone turbulence in a spilling breaker, Coastal Engineering, 27, 131-160.

Veron, F., Melville, W.K. and Lenain, L. 2008. Infrared techniques for measuring ocean surface processes, Journal of Atmospheric and Oceanic Technology, 25, 307-326. 
Watanabe, Y. and Mori, N. 2008. Infrared measurements of surface renewal and subsurface vorticies in nearshore breaking waves, Journal of Geophysical Research-Oceans, 113, C07015.

Watanabe, Y., Saeki, H.S. and Hosking, R.J. 2005. Three-dimensional vortex structures under breaking waves, Journal of Fluid Mechanics, 545, 291-328. 